\title{
The stratified Boycott effect
}

\author{
By TOM PEACOCK, FRANCOIS BLANCHETTE \\ AND JOHN W. M. BUSH
}

Department of Mathematics, Massachusetts Institute of Technology, Cambridge, MA 02219, USA

(Received 18 November 2002 and in revised form 1 November 2004)

We present the results of an experimental investigation of the flows generated by monodisperse particles settling at low Reynolds number in a stably stratified ambient with an inclined sidewall. In this configuration, upwelling beneath the inclined wall associated with the Boycott effect is opposed by the ambient density stratification. The evolution of the system is determined by the relative magnitudes of the container depth, $h$, and the neutral buoyancy height, $h_{n}=c_{0}\left(\rho_{p}-\rho_{f}\right) /|\mathrm{d} \rho / \mathrm{d} z|$, where $c_{0}$ is the particle concentration, $\rho_{p}$ the particle density, $\rho_{f}$ the mean fluid density and $\mathrm{d} \rho / \mathrm{d} z<0$ the stable ambient stratification. For sufficiently weak stratification, $h<h_{n}$, the Boycott layer transports dense fluid from the bottom to the top of the system; subsequently, the upper clear layer of dense saline fluid is mixed by convection. For sufficiently strong stratification, $h>h_{n}$, layering occurs. The lowermost layer is created by clear fluid transported from the base to its neutral buoyancy height, and has a vertical extent $h_{n}$; subsequently, smaller overlying layers develop. Within each layer, convection erodes the initially linear density gradient, generating a step-like density profile throughout the system that persists after all the particles have settled. Particles are transported across the discrete density jumps between layers by plumes of particle-laden fluid.

\section{Introduction}

The development of horizontal layers, between which discrete changes in fluid properties arise, is a ubiquitous feature of double-diffusive systems (Brandt \& Fernando 1994). Much of the work in this area has focused on thermohaline systems relevant in the oceans, but double-diffusive systems also arise in a variety of geological systems and industrial processes. Double-diffusive layers may arise through salt fingering, overstability (Turner 1985) and when a stable salinity gradient is heated from below (Turner 1968; Huppert \& Linden 1979). Experiments on sidewall heating indicate the emergence of small-scale layering at onset (Thorpe, Hutt \& Soulsby 1969; Tanny \& Tsinober 1988); however, a sequence of layer mergings leads ultimately to a steady state in which the layer size scales as $\Delta \rho_{T} /|\mathrm{d} \rho / \mathrm{d} z|$, where $\mathrm{d} \rho / \mathrm{d} z<0$ is the stable initial ambient stratification and $\Delta \rho_{T}$ is the lateral density difference owing to temperature (Chen, Briggs \& Wirtz 1971; Huppert \& Turner 1978, 1980; Narusawa \& Suzukawa 1981; Suzukawa \& Narusawa 1982; Huppert, Kerr \& Hallworth 1984). Theoretical investigations into the origins of layers that arise from sidewall heating or cooling reveal both shear and sideways diffusive instabilities (Thorpe et al.1969; Hart 1971, 1973; Paliwal \& Chen 1980b; Thangam, Zebib \& Chen 1981; Kerr 1989, 2000).

The presence of a sloping boundary provides another means of generating horizontal layers in a double-diffusive system. For fluid stably stratified by a single component, convection develops beneath the inclined wall owing to the no-flux 
requirement at the boundary (Phillips 1970; Wunsch 1970). If the system is stratified with two components of markedly different diffusivity, one component being stably stratified and the other unstably stratified, such that the net stratification is stable, convection that develops beneath the wall owing to the faster diffusing component is opposed by the ambient density stratification; consequently, a series of intrusions proceed into the ambient from the underside of the wall. Turner (1974), Turner \& Chen (1974) and Linden \& Weber (1977) investigated this phenomenon experimentally; supporting theoretical studies were undertaken by Chen (1975) and Paliwal \& Chen $(1980 a, b)$. In this paper, we report the analogous layering that arises from sedimentation in a salt stratified ambient with an inclined sidewall, wherein particles assume the role of the faster diffusing component in generating upflow beneath the sloping wall.

The ability of particles to play the role of a diffusing component in a doublediffusive system has been well-established. Green (1987) examined the convective instability arising in a stably stratified system marked by hot particle-laden fluid overlying cold fluid, and explained the resulting millimetre-scale plumes by analogy with salt fingering (Schmitt 1994). The development of layering in a polydisperse suspension was reported by Brewer (1884), and explained in terms of the interaction of sidewall heating with the density stratification arising from the differential settling of polydisperse particles (Mendenhall \& Mason 1923). Siano (1979) and Mueth et al. (1996) performed further experimental investigations into the phenomenon, and a detailed numerical study was undertaken by Hosoi \& Dupont (1996).

The Boycott effect generates convection when an inclined upper surface is introduced into a suspension of heavy particles. The particles settle under the influence of gravity, thereby creating a layer of particle-free fluid beneath the sloping surface. The clear fluid within the thin layer is buoyant and so ascends beneath the sloping wall. The tangential gradient in flux of clear fluid along the wall requires that fluid be drawn from the suspension into the particle-free layer. The resulting drag balances the gravitational force on the particles adjoining the Boycott layer; in equilibrium, there exists a steady boundary layer whose thickness grows with distance along the wall. The effect was first reported by Boycott (1920), who noted the anomalously fast settling of red blood cells in a container with sloping boundaries. A heuristic model for the descent rate of the suspension was put forward by Ponder (1925) and Nakamura \& Kuroda (1937). Hill, Rothfus \& Li (1977), Acrivos \& Herbolzheimer (1979) and Leung \& Probstein (1983) presented more complete theoretical descriptions and accompanying experimental validation. Their analysis indicates that the thickness, $\delta$, and velocity, $u$, of the Boycott layer scale as

$$
\delta \sim \Lambda^{-1 / 3} l, \quad u \sim \Lambda^{1 / 3} v_{0},
$$

where $\Lambda=(9 / 2)(l / a)^{2} c_{0}, l$ is a characteristic length scale (typically the depth of the suspension), $a$ the particle radius, $c_{0}$ the particle volume fraction and $v_{0}$ the hindered settling speed of the particles. Furthermore, Acrivos \& Herbolzheimer (1979) showed that to account for the influx of fluid into it, the Boycott layer grows as $\delta \sim x^{1 / 3}$, where $x$ is the coordinate along the inclined wall.

Here, we investigate the influence of a stably stratified ambient on the Boycott effect. The presence of a suspension in a stratified ambient bound in a non-rectangular geometry may arise in a variety of geophysical settings. One example is beneath ice shelves in the ocean, where a stable salinity or temperature gradient exists, and particles are present in the form of suspended sediment or algae. An analogous setting may arise at the upper sloping surface of the subterranean Antarctic Lake Vostok, where 


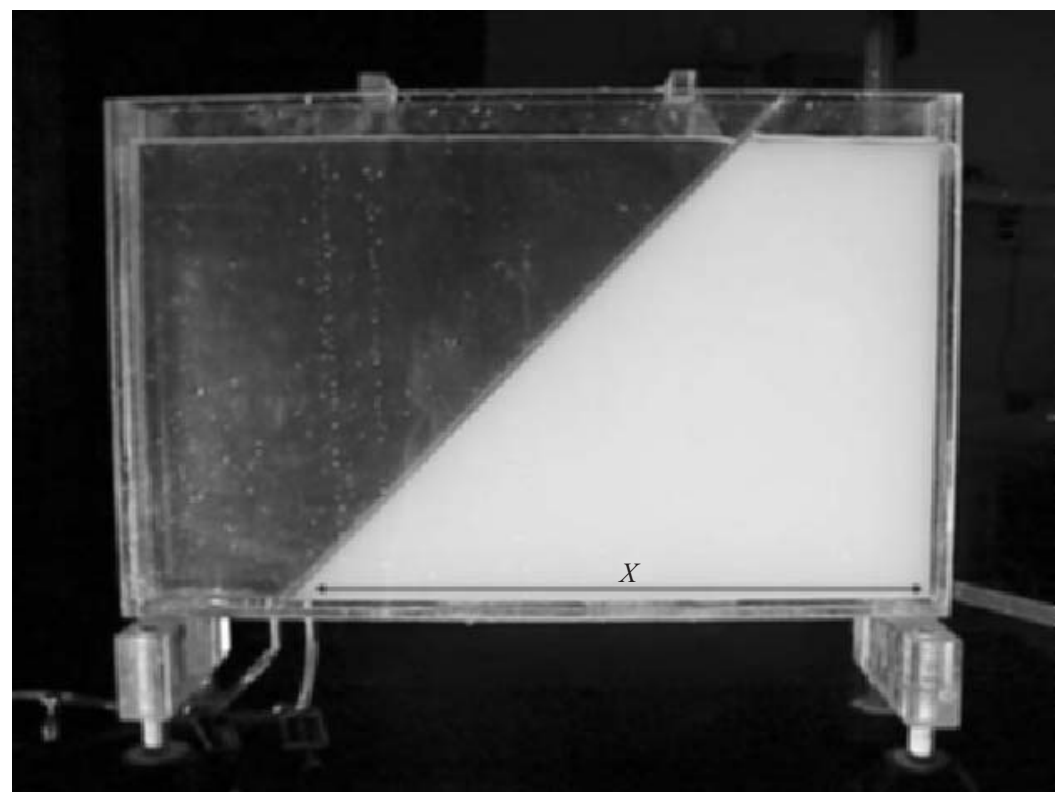

Figure 1. The experimental apparatus. The dimensions of the tank are $40 \mathrm{~cm} \times 25 \mathrm{~cm} \times 2 \mathrm{~cm}$. A sloping wall is introduced at an angle of $45^{\circ}$, across the centre. In this image, the right-hand side of the tank is filled with sediment-laden water and the left-hand side with clear water. The length of the base section $X$ is $32.2 \mathrm{~cm}$.

particles are believed to be released by melting (Wuest \& Carmack 2000). Finally, in magma chambers, compositional and thermal gradients may generate a stable density stratification and cooling generates particulate matter in the form of negatively buoyant crystals, or buoyant bubbles exsolving from the bulk (Turner 1980).

We anticipate that the evolution of the Boycott layer in a stably stratified ambient will be determined by the relative sizes of the depth of the ambient $h$ and the neutral buoyancy height

$$
h_{n}=\frac{\Delta \rho_{p}}{|\mathrm{~d} \rho / \mathrm{d} z|},
$$

defined in terms of the density difference due to particles $\Delta \rho_{p}=c_{0}\left(\rho_{p}-\rho_{f}\right)$, the particle density $\rho_{p}$ and the mean fluid density $\rho_{f}$. If $h<h_{n}$, we expect that the Boycott layer will simply serve as a mechanism for transporting dense fluid from the bottom to the top, and so mixing the system. If $h>h_{n}$, we expect the upflow within the Boycott layer to be disrupted, giving rise to layers of characteristic size $h_{n}$.

To test this physical picture, we performed a series of experiments in which particles settled in a salt stratified ambient. In $\S 2$, we describe the experimental techniques, and in $\S 3$, provide a detailed presentation of our experimental results. The study is reviewed in the context of related double-diffusive flows in $\S 4$.

\section{Experimental technique}

A photograph of the experimental apparatus is presented in figure 1 . The experiment comprised a Perspex tank $25 \mathrm{~cm}$ high, $40 \mathrm{~cm}$ long and $2.5 \mathrm{~cm}$ wide, with vertical sidewalls. The top was open and the sidewalls slotted to facilitate the introduction of a sloping wall at an angle of $45^{\circ}$ across the centre of the tank. Vibration control mounts 
were used to level the system and isolate the tank from mechanical disturbances. Holes were drilled in the base of the tank so that it could be filled from below.

Two different configurations were used in the experiments. The first configuration involved fine silica particles of density $2.65 \mathrm{~kg} \mathrm{~m}^{-3}$ settling in stratified salt water. The particles were obtained by differential settling from a sample of MinUSil 10, obtained from US Silica. Using the Richardson-Zaki (1954) formulation for the hindered settling function, the particle radius was estimated to be $4.1 \pm 0.8 \mu \mathrm{m}$. The corresponding Stokes settling speed of the particles was $65 \pm 25 \mu \mathrm{m} \mathrm{s}^{-1}$. The volume fraction of particles used was typically $0.3 \%$, giving an added density due to particles of $\Delta \rho_{p}=5 \pm 0.2 \mathrm{~kg} \mathrm{~m}^{-3}$. The second configuration involved MO-SCI GL-0191 glass spheres of radius $17.5 \pm 1.5 \mu \mathrm{m}$ settling in a salt stratified solution comprising $63 \%$ glycerol and $37 \%$ water. In the absence of salt, the kinematic viscosity of this mixture was $12.15 \mathrm{cS}$, which gave a settling speed of approximately $88 \pm 15 \mu \mathrm{m} \mathrm{s}^{-1}$. The presence of salt increased the viscosity of the solution significantly, giving a kinematic viscosity in excess of $21 \mathrm{cS}$ for salt saturated solutions. To minimize the influence of this variable viscosity, only weak salinity gradients were used in the second configuration.

The tank was filled from below, and a linear salt stratification established using the double bucket technique (Oster 1965). One bucket was filled with saline solution and a known volume fraction of particles. The other contained fresh solution with the same volume fraction of particles. In this manner, it was possible to establish an initial state characterized by a stable linear salinity gradient and a uniform particle distribution. The filling process took four minutes, which was short compared to the time taken for particles to settle across the height of the container (approximately one hour). A PME salinity probe mounted on a translation stage measured the resulting density gradient, which was initially linear. The probe was calibrated using an Anton Parr DMA 35 densitometer that measured density to an accuracy of $0.2 \mathrm{~kg} \mathrm{~m}^{-3}$. Once the tank was filled, the sloping wall was introduced; this process took approximately $4 \mathrm{~s}$ and was sufficiently slow that disturbances to the ambient fluid were minimized, but fast compared to the time taken for the Boycott layer to be established (about $10 \mathrm{~s}$ ). Indeed, the time taken for the introduction of the wall was varied between 1 and $10 \mathrm{~s}$ without affecting results. After the wall was introduced, a clear layer developed in which particle-free fluid rose beneath the inclined surface. The evolution of the system was found to depend strongly on the ratio $h / h_{n}$, in a manner to be detailed in $\S 3$.

\section{Experimental results}

We consider in turn the two distinct flow regimes that arise according to the relative magnitudes of the layer depth $h$ and the level of neutral buoyancy $h_{n}$ defined in (1.2).

\subsection{Weak salinity gradient}

For $h<h_{n}$, clear fluid was transported upwards within a Boycott layer of characteristic thickness $2.5 \mathrm{~mm}$ with a characteristic speed of $0.5 \mathrm{~mm} \mathrm{~s}^{-1}$, values consistent with the theory of Acrivos \& Herbolzheimer (1979), and the thickness of the Boycott layer was clearly seen to increase with distance along the wall. Consequently, a sharp interface between clear fluid and suspension descended from the top of the tank. The evolution of the position of the clear fluid interface was measured for the $17.5 \pm 1.5 \mu \mathrm{m}$ radius glass spheres settling in a glycerol-water solution, and the results are presented in figure 2. The solid line corresponds to the descent rate expected for particles of radius $16 \mu \mathrm{m}$ (the smallest present in our sample) in a homogeneous ambient. This line was 


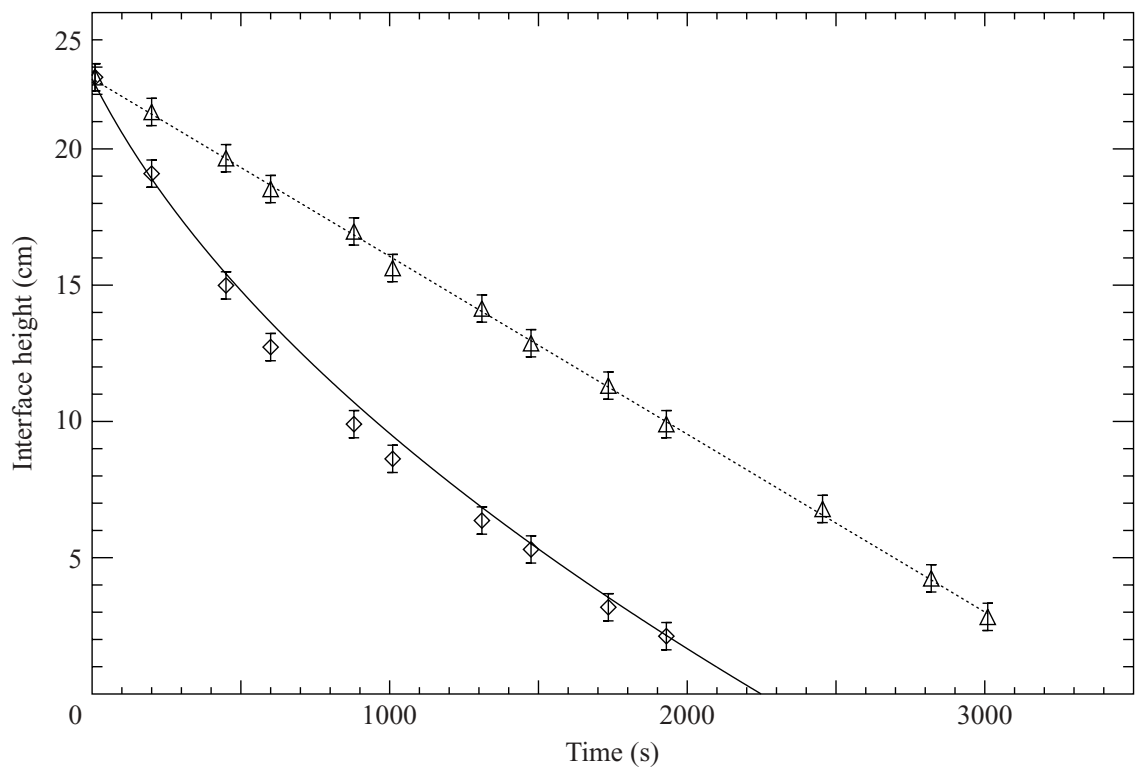

FIGURE 2. The height of the interface as a function of time for $h / h_{n}=0.5$. $\diamond$, Boycott effect; experiment. $\triangle$, no Boycott effect; experiment. The experimental data points were obtained by measuring the height of the interface on the left- and right-hand sides of the sloping wall in figure 3 . The solid and dashed lines are the corresponding theoretical curves.

determined for our trapezoidal geometry using the theory proposed by Ponder (1925) and Nakamura \& Kuroda (1937) for a homogeneous ambient, which predicts that the evolution of the interface height, $H(t)$, is governed by

$$
\frac{\mathrm{d} H}{\mathrm{~d} t}(X-H)=-v_{0} X,
$$

$X$ being the length of the base of the tank (see figure 1). The dotted line indicates the descent rate of the interface expected for particles in the absence of the Boycott effect: $\mathrm{d} H / \mathrm{d} t=-v_{0}$. From the evolution of the suspension on the opposite side of the sloping wall (see figure 3), this settling rate was readily verified.

The clear fluid transported from the base of the tank mixed with that already above the suspension. Since the density of clear fluid transported from the base of the tank decreases with time, we anticipate the development of a weak stratification in the clear upper layer. The evolution of the clear fluid interface and the corresponding salinity profiles are presented in figure 3 . Note that the time taken between obtaining a salinity gradient and the corresponding image was approximately one minute (required for complete immersion and withdrawal of the salinity probe). At time $t=0$, the interface is at the top of the tank and the density profile linear throughout; the apparent smallscale deviations from linearity are due to the combination of high probe sensitivity, the weak salinity gradient, and disturbances created by the probe's motion through the ambient. After $t=5 \mathrm{~min}$ the profile is linear within the suspension, but irregular above the clear fluid interface because of mixing; such intermediate stages are marked by clear salty fluid overlying less saline suspension. After $40 \mathrm{~min}$, particles have settled out completely and a relatively weak stratification persists.

The final density profile arising cannot be simply predicted owing to the heightened mixing in the upper clear fluid, prompted by the salinity jump at the interface 
(a)

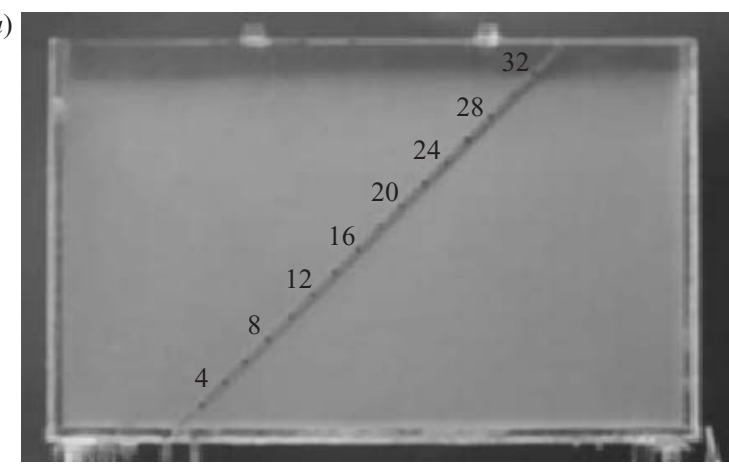

(b)

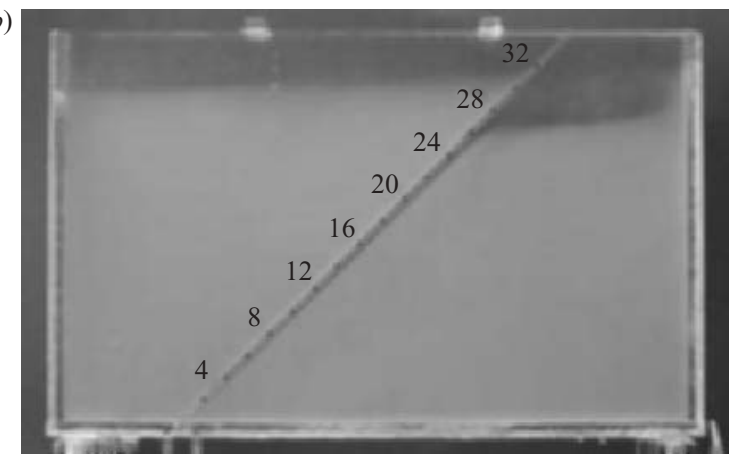

(c)

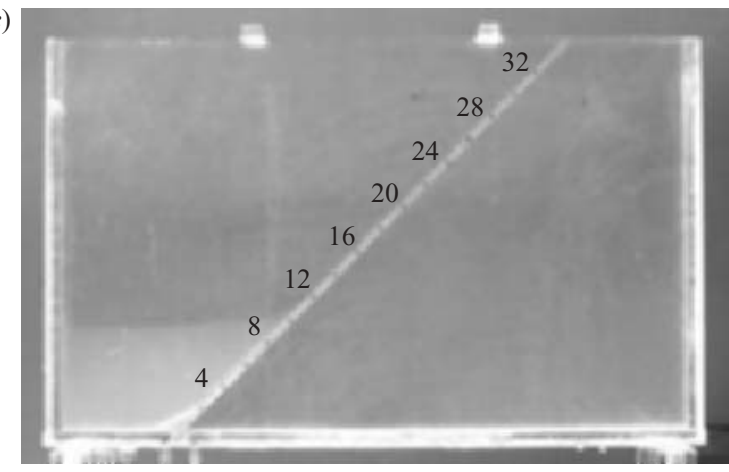

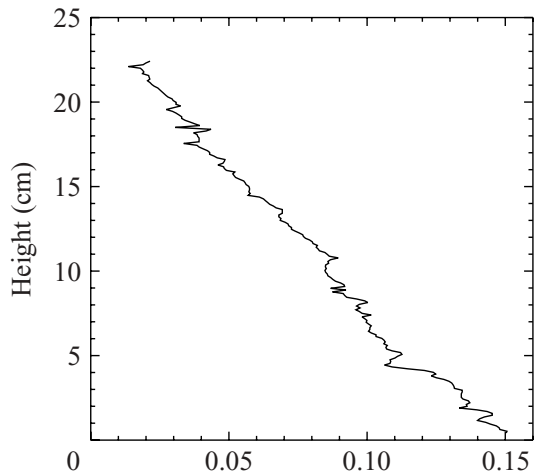
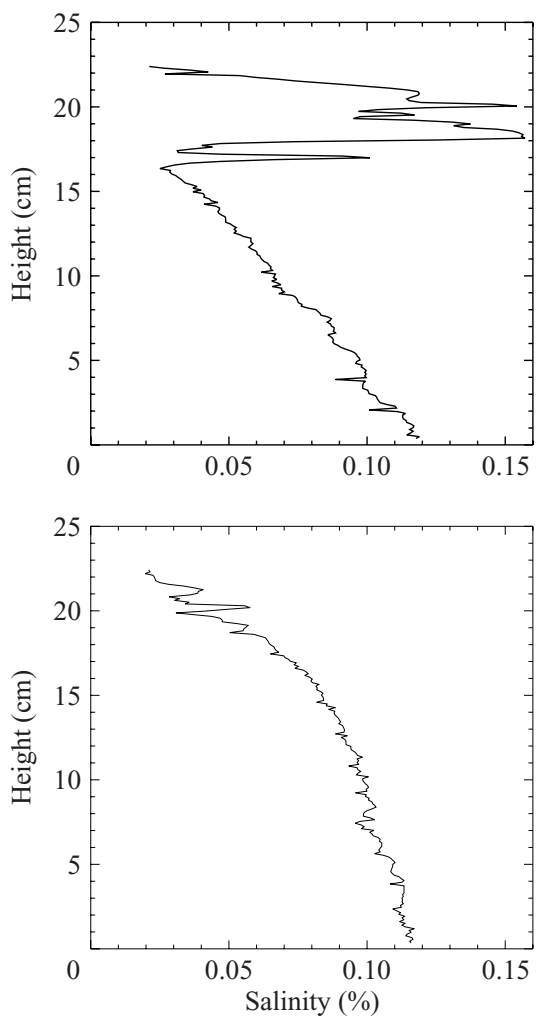

FIGURE 3. Images and salinity profiles for the stratified Boycott effect for $h / h_{n}=0.5$. Salinity profiles were measured approximately $8 \mathrm{~cm}$ from right-hand wall. (a) $t=0 \mathrm{~min}$. $(b) t=5 \mathrm{~min}$. (c) $t=40 \mathrm{~min}$. The settling speed of particles in the absence of the Boycott effect can be determined from the interface on the left of the sloping wall.

between clear fluid and suspension. As the interface descended, buoyant clear fluid was released and rose in the form of millimetric plumes. The plumes were visible by virtue of particles that they entrained from the suspension. A schematic illustration of the plumes is presented in figure 4. A related scenario arising with a polydisperse suspension has been considered by Kerr (1991b) and Huppert, Kerr \& Lister (1991), who deduced the influence of the associated plume-induced mixing on the descent rate of the suspension. In our system, such mixing was negligible, as evidenced by the 


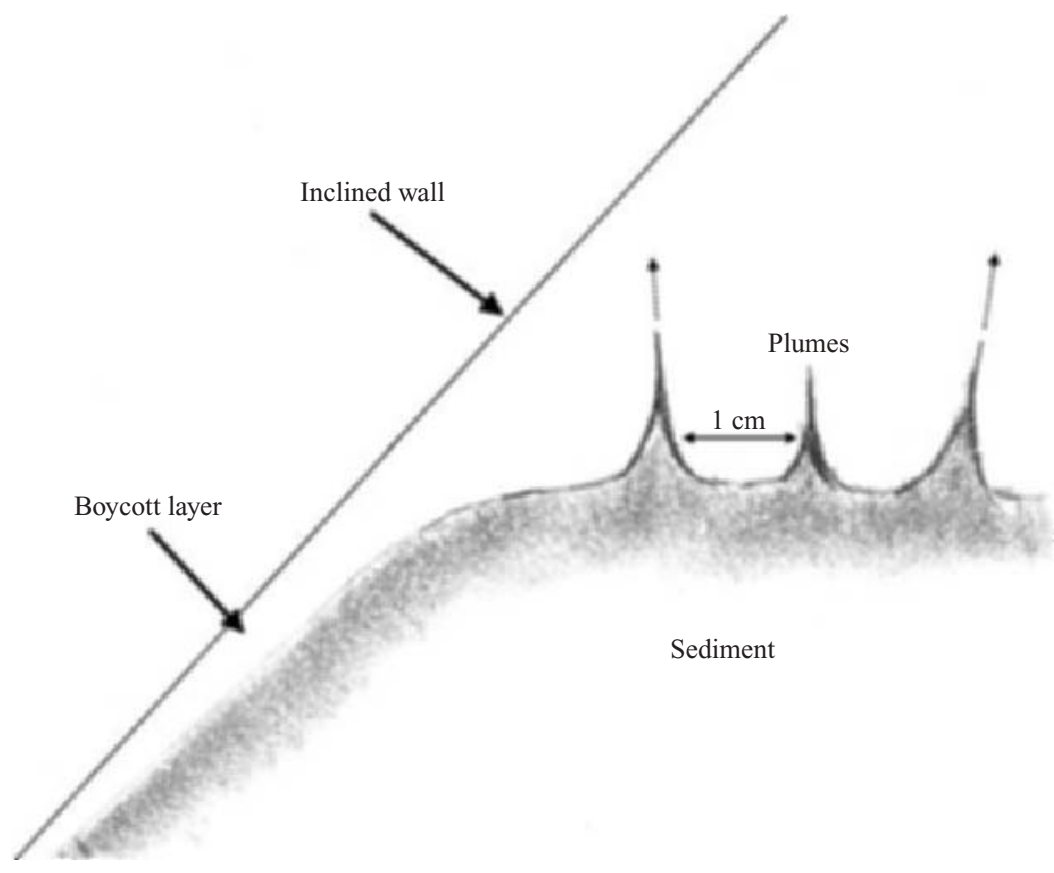

FigURE 4. A sketch of buoyant particle plumes at the upper interface between clear fluid and suspension. The plumes consist of buoyant fluid released by the settling of the interface and some entrained particles. Microplumes are also evident in the multilayer case $h>h_{n}$.

good agreement between the descent rate of the suspension and that predicted for the Boycott effect in a homogeneous ambient (figure 2).

\subsection{Strong salinity gradient}

For $h>h_{n}$, the upflow within the Boycott layer stalled, giving rise to intrusions into the stratified ambient. The development of the first and lowermost, hereinafter referred to as the 'primary', intrusion was followed by a series of overlying secondary intrusions that appeared simultaneously. The experiments described in this section were performed using $4.1 \pm 0.8 \mu \mathrm{m}$ particles settling in salt water. The characteristic thickness of the Boycott layer was $1 \mathrm{~mm}$ and the characteristic flow speed within the layer was $2 \mathrm{~mm} \mathrm{~s}^{-1}$. The increase in speed and decrease in thickness relative to the previous experiments is consistent with the scaling (1.1).

We first performed a series of experiments in order to ascertain the dependence of the primary intrusion height, $h_{p}$, on the density gradient. For this study, the primary intrusion height was defined to be the height at which clear fluid from the Boycott layer first intruded into the ambient. The density gradient was initially varied in the range -20 to $-300 \mathrm{~kg} \mathrm{~m}^{-4}$ for a constant volume fraction of silica particles. The added density due to the presence of particles was $\Delta \rho_{p}=5 \pm 0.2 \mathrm{~kg} \mathrm{~m}^{-3}$, giving $1<h / h_{n}<15$. The results are presented in figure 5 , where it is evident that the height of the primary intrusion decreases monotonically with increasing density gradient. The solid line drawn through the results is the neutral buoyancy curve (1.2). The dependence of $h_{p}$ on the particle volume fraction was also systematically investigated. The experiments were performed at an ambient stratification of $-57 \pm 2 \mathrm{~kg} \mathrm{~m}^{-4}$ for four different particle volume fractions: $\Delta \rho_{p}=2.5,5.0,7.5$, and $10.0 \pm 0.2 \mathrm{~kg} \mathrm{~m}^{-3}$. The anticipated dependence (1.2) is again supported well by the data, as is evident in figure 6 . 


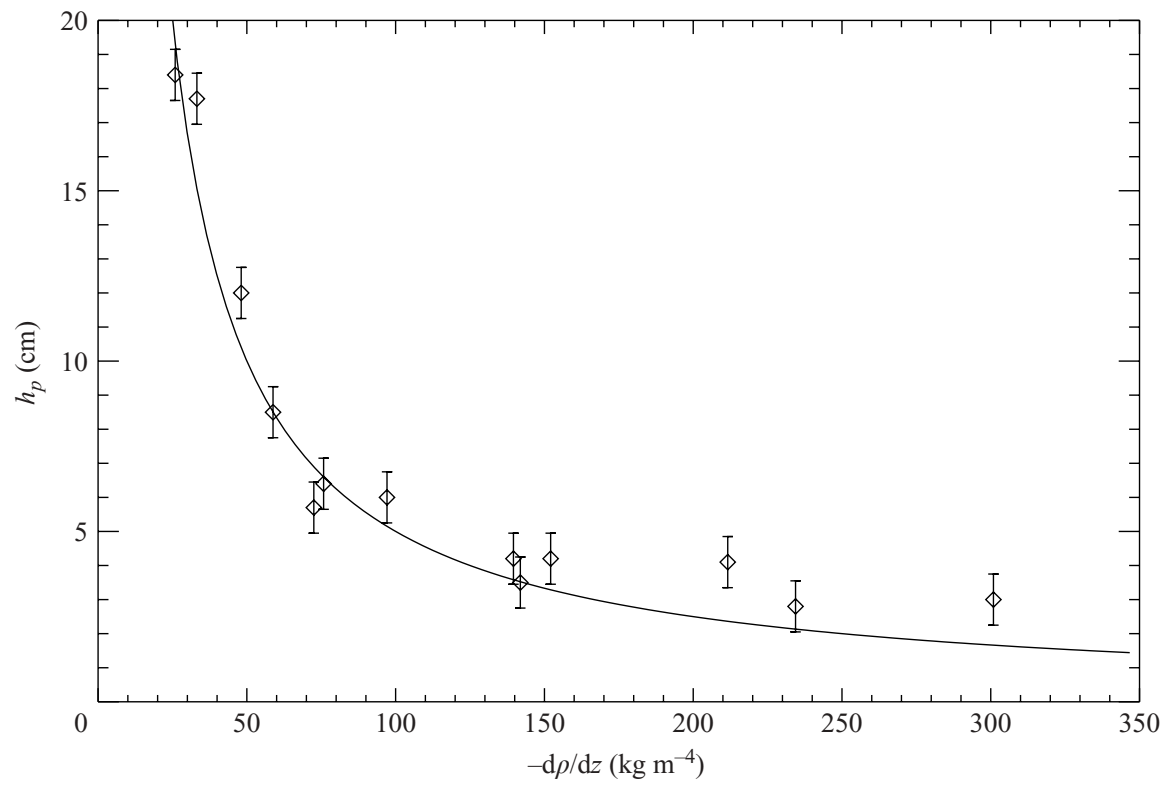

FIGURE 5. Experimental results $(\diamond)$ for the dependence of the primary intrusion height $h_{p}$ on the ambient stratification. The solid curve drawn on the graph is the neutral buoyancy curve given in (1.2). Error bars are shown.

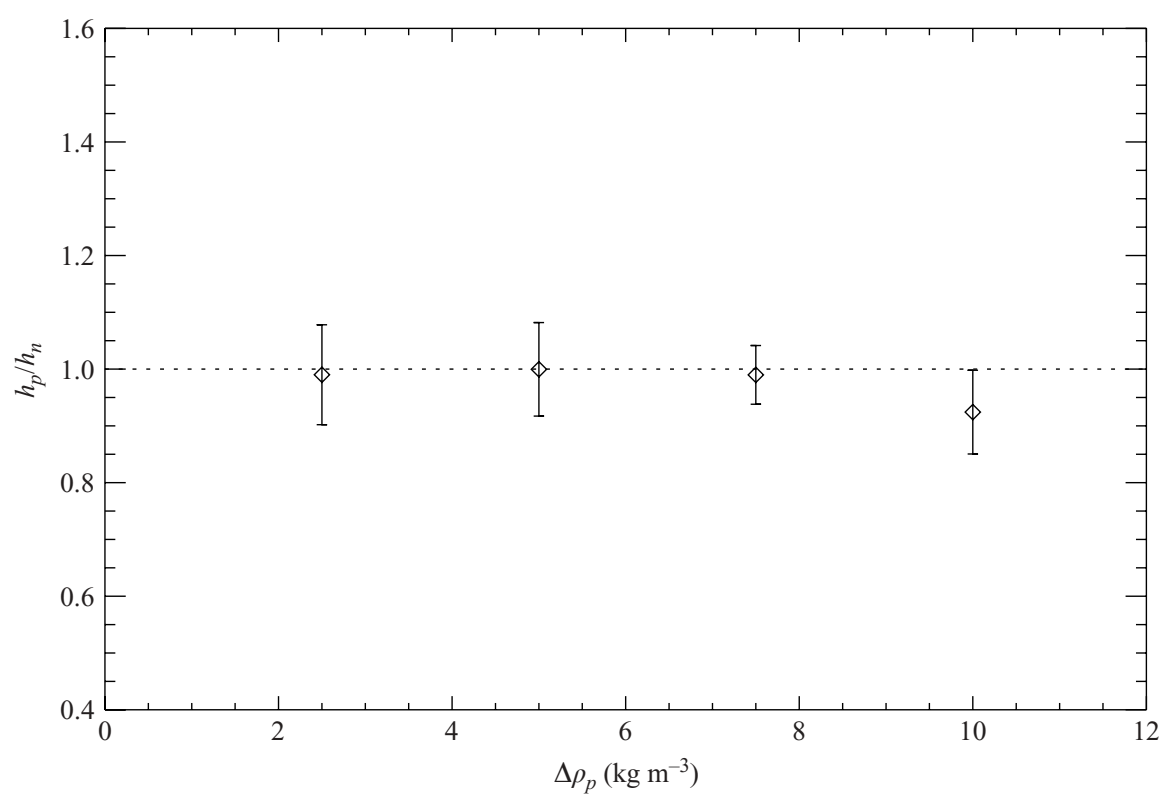

FIGURE 6 . The ratio of the primary intrusion height $h_{p}$ to the neutral buoyancy height $h_{n}$ (defined in (1.2)) as a function of the added density due to particles, $\Delta \rho_{p}$. The results were obtained for a density gradient of $-57 \mathrm{~kg} \mathrm{~m}^{-4}$.

As the system evolved in this strong stratification limit, a layered structure developed throughout the fluid domain, on a timescale of several minutes. Each layer was a convection cell characterized by an intrusion at the top and a return flow within the 
(a)

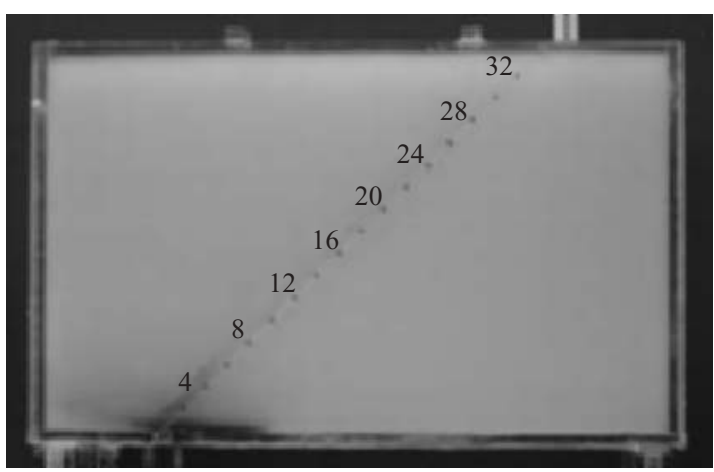

(b)

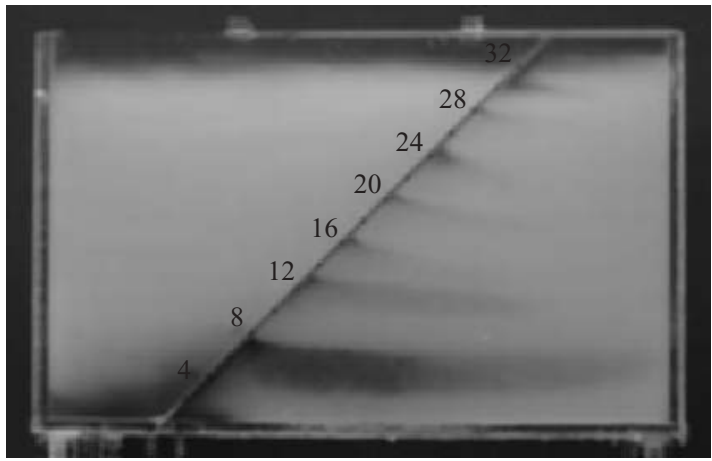

(c)

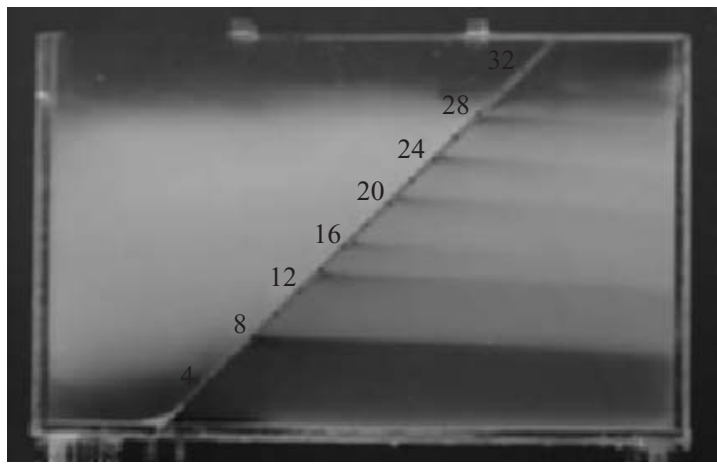

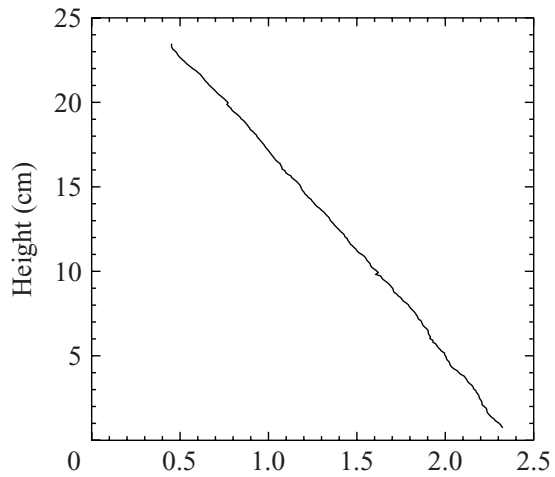
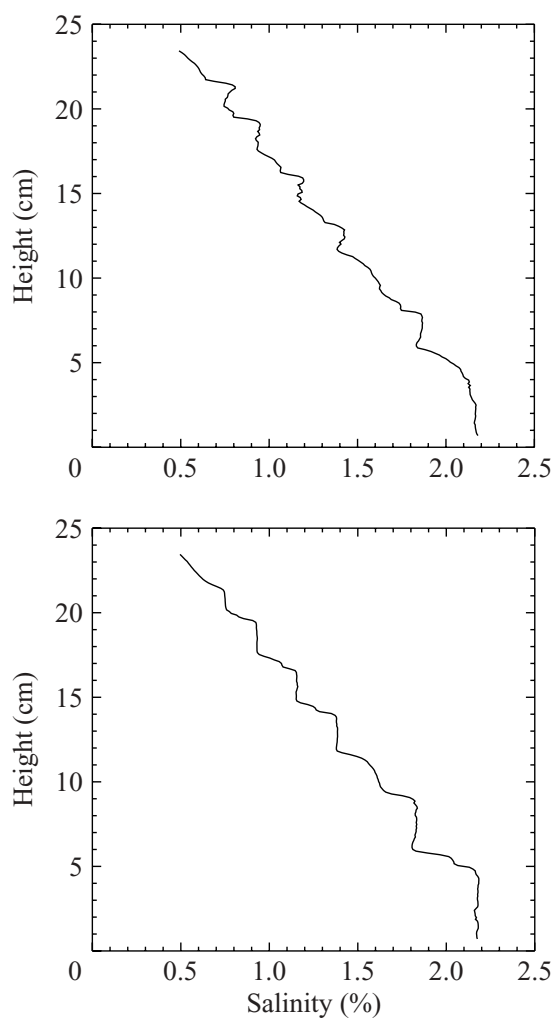

FIGURE 7. Images of layering arising for a strong salinity gradient $\left(h / h_{n}=3.85\right)$ and corresponding salinity profiles taken at a distance of approximately $8 \mathrm{~cm}$ from the right-hand wall (the probe can be seen near the top right-hand corner of $(a)$ ). Note the larger primary layer at the base of the tank and the smaller overlying secondary layers. $(a) t=0$ min. (b) $t=3 \mathrm{~min}$. (c) $t=10 \mathrm{~min}$. The salinity profile in $(c)$ remained once all the particles had settled.

body of the layer. This fluid motion is rendered visible in figures 7 and 8 through the introduction of dye. First, a small amount of finely ground potassium permanganate was introduced near the base of the inclined wall. From the images presented in figure 7, which were obtained for $\mathrm{d} \rho / \mathrm{d} z=-77 \pm 1 \mathrm{~kg} \mathrm{~m}^{-4}$, we can see that dyed fluid from the base of the tank rose no further than the primary intrusion height, where it intruded horizontally into the ambient. The convection within each layer is 
(a)

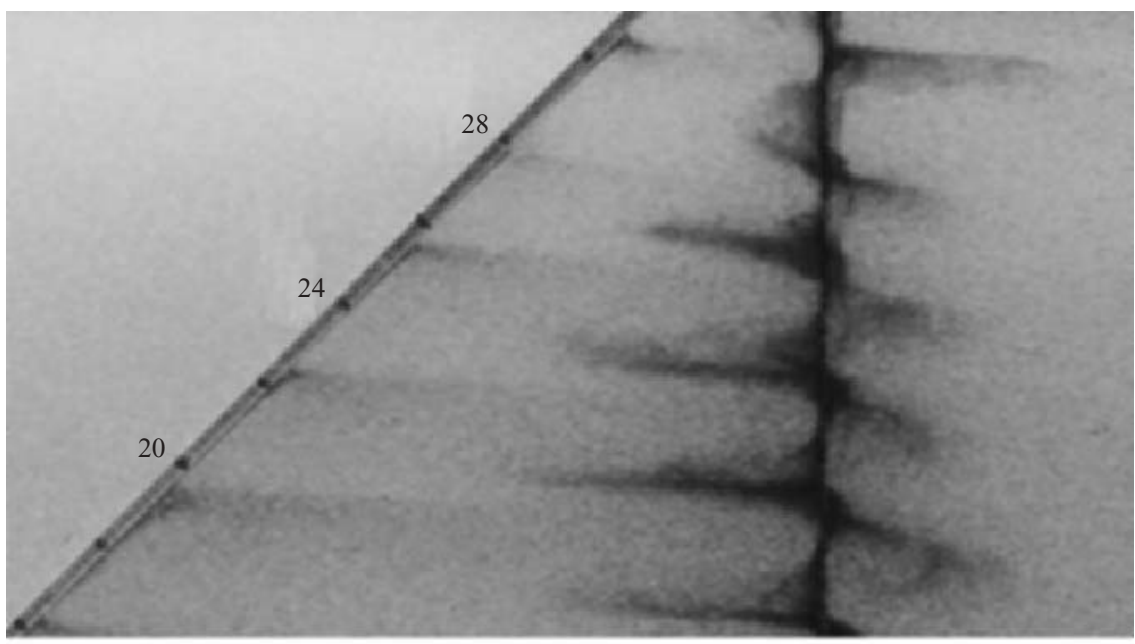

(b)

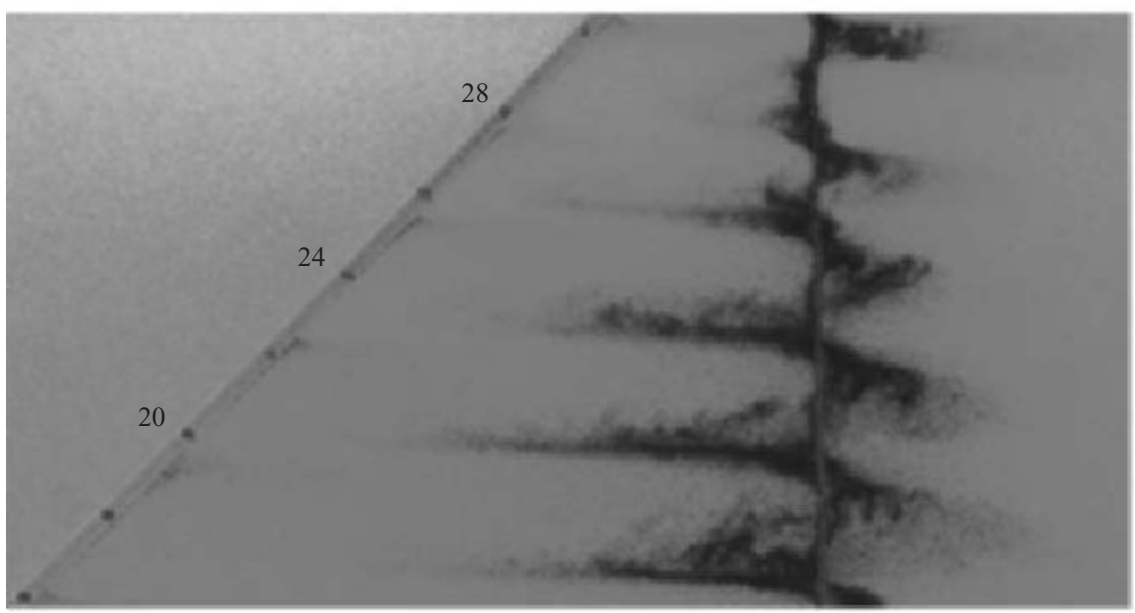

(c)

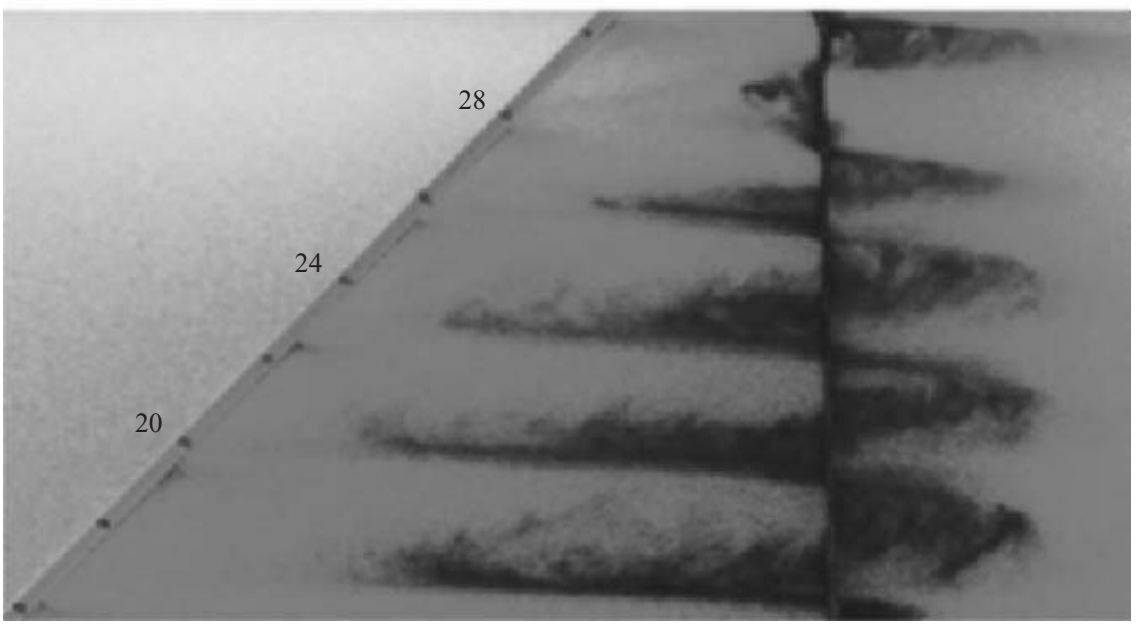

FIGURE 8. A time sequence illustrating the advection of dye released from a vertical thread for the case of strong stratification, $h>h_{n}$. The images were taken 1 min apart. 


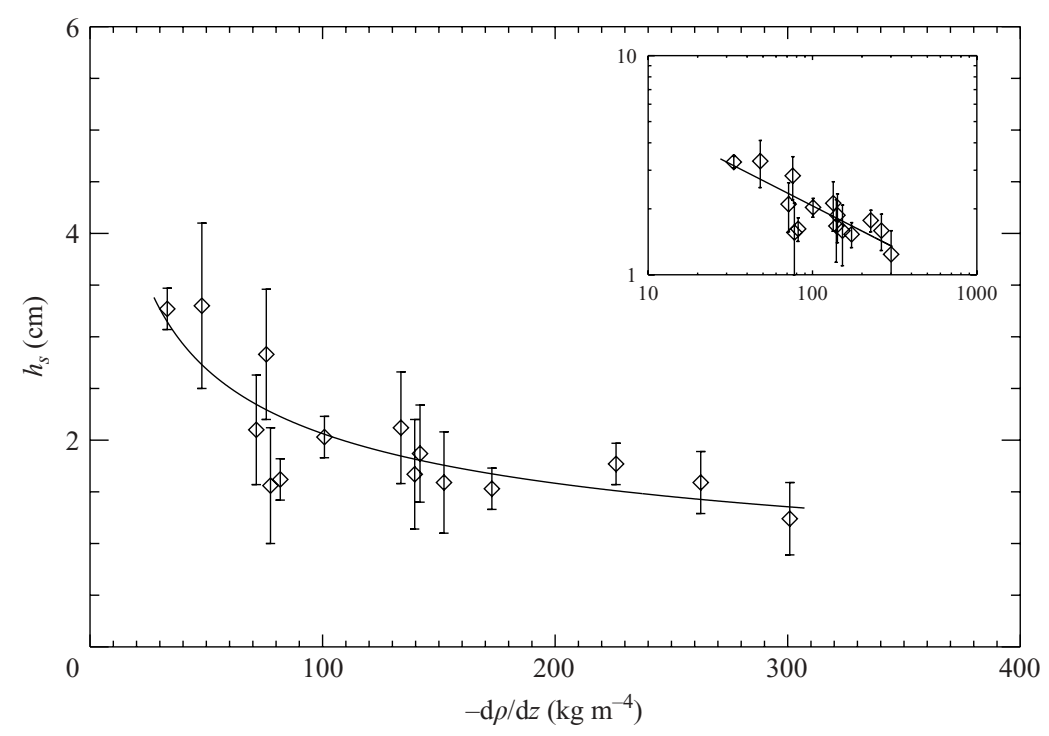

FiguRE 9. Experimental results for the dependence of the mean height of the secondary intrusions on the ambient stratification. Inset: equivalent $\log -\log$ plot. The best-fit line to the data on the log-log plot has slope 0.38; the corresponding line has been drawn through the experimental results in the main plot.

visualized in figure 8 through the transport of dye released from a vertical thread. This convection served to mix fluid within each layer, eroding the initially linear density gradient and generating a step-like density profile, as is evident in the salinity profiles in figures $7(b)$ and $7(c)$. When dye was introduced at an intermediate height in the tank, it remained trapped within a single layer, indicating that fluid was not exchanged between layers. The layering process was not entirely steady, and layers occasionally merged or split on a timescale of minutes. After all the particles had settled, the step-like density profile in figure $7(c)$ persisted until ultimately eroded through diffusion.

The secondary intrusions were recognizable as disturbances emerging simultaneously from the Boycott layer soon after the development of the primary intrusion; these are evident above the primary intrusion in figure 7. To investigate these intrusions, we used density gradients in the range -40 to $-300 \mathrm{~kg} \mathrm{~m}^{-4}$, corresponding to $2<h / h_{n}<15$ for $\Delta \rho_{p}= \pm 0.2 \mathrm{~kg} \mathrm{~m}^{-3}$. The observed dependence of mean secondarylayer height on stratification is reported in figure 9. The secondary layers, of size $h_{s}$, are smaller than their primary counterparts. Furthermore, the scale of the secondary layers is not inversely proportional to the density gradient of the ambient as we would anticipate from (1.2). Rather, a best-fit line to the results on a logarithmic plot gives the scaling

$$
h_{s} \sim\left(\frac{\mathrm{d} \rho}{\mathrm{d} z}\right)^{-0.38} .
$$

Observations also indicated that the secondary-layer size increased with increasing particle volume fraction. For example, for a density gradient of $\mathrm{d} \rho / \mathrm{d} z=-150 \mathrm{~kg} \mathrm{~m}^{-4}$, the layer size increased from approximately $2 \mathrm{~cm}$ to $2.8 \mathrm{~cm}$ as $\Delta \rho_{p}$ was increased from $5 \mathrm{~kg} \mathrm{~m}^{-3}$ to $16 \mathrm{~kg} \mathrm{~m}^{-3}$. 


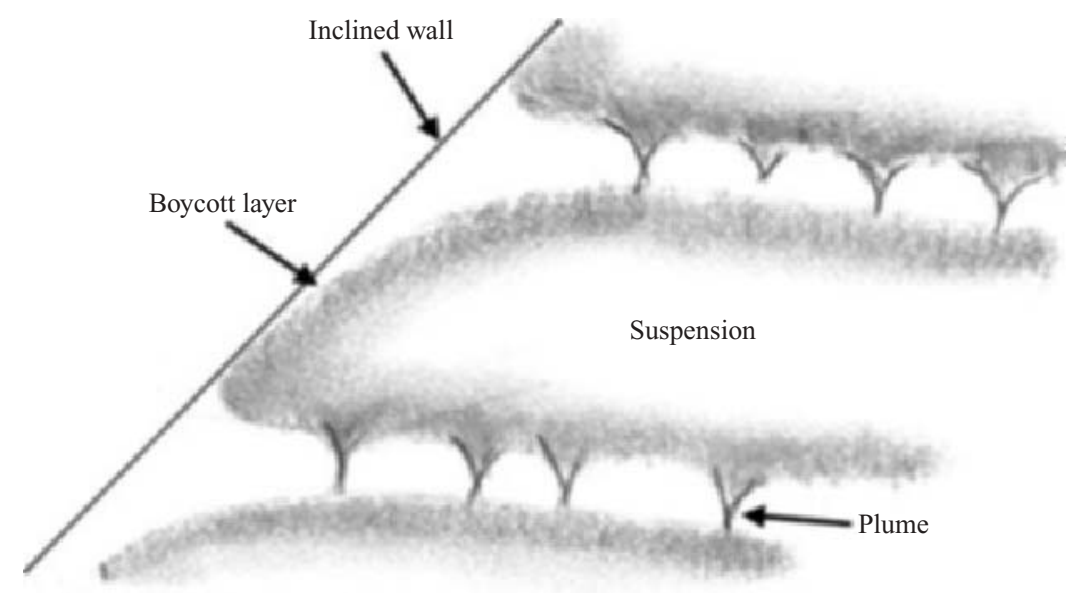

FIGURE 10. A sketch of the negatively buoyant particle plumes at the interface between layers. The characteristic spacing of the plumes was approximately $1 \mathrm{~cm}$.

The layers were initially tilted downwards, as is apparent in figure $7(b)$. The origin of this tilting is a secular decrease in the density of fluid within the Boycott layer. The height of the point of intrusion beneath the inclined wall increased with time, indicating that the density of clear fluid within the Boycott layer necessarily decreased with time, thus giving rise to an apparent tilting of the intrusion. The secular decrease in density is a consequence of mixing within a layer, which serves to reduce the salinity near the base of the layer, from which much of the clear fluid is drawn. After the layering has evolved for a sufficient time, the density within a layer becomes uniform and tilting is no longer apparent (figure $7 c$ ). Note that this mechanism for tilting is distinct from that observed in thermohaline systems (Huppert \& Turner 1980).

The vertical transport of particles between layers was not through uniform settling. Rather, as a consequence of the density jump between layers, millimetric particleladen plumes developed at the interfaces and penetrated downwards through the clear fluid interfaces (figure 10). The generation of microplumes through sedimentation across a density interface has been considered by Hoyal, Bursik \& Atkinson (1999) and Parsons, Bush \& Syvitski (2001), and is influenced by the increase of particle concentration required by conservation of particle flux across the interface. It was difficult to obtain a quantitative measure of the overall settling time for the system, as there was no readily discernible interface separating suspension from particle-free fluid. However, it was clear that particle settling was significantly impeded by the presence of the layering; for example, when the Boycott settling time in a homogeneous ambient was approximately $37.5 \mathrm{~min}$, suspension persisted for more than $1 \mathrm{~h}$ in the stratified environment.

\section{Discussion}

We have performed a careful set of experiments in order to elucidate the influence of ambient stratification on the Boycott effect. Two distinct regimes exist according to the relative magnitudes of $h$ and $h_{n}$, as defined in (1.2). For a weak salinity gradient, $h<h_{n}$, the Boycott layer transports dense fluid from the base of the tank to the top, where it mixes with clear fluid. The concomitant overturning erodes the initially linear density gradient; once all the particles have settled, the density gradient is reduced 
throughout the system. The presence of a salinity jump at the sediment interface generates plumes as a result of settling, a phenomenon investigated by Kerr (1991b) and Huppert et al. (1991). In our study, particle resuspension by the plumes had little effect on the descent of the interface; the timescale of settling in the weak salinity limit was that expected for the Boycott effect in a homogeneous ambient.

For a strong salinity gradient, $h>h_{n}$, the Boycott layer stalls under the influence of gravity, giving rise to a series of distinct layers. A primary layer with the expected vertical extent set by (1.2) appears when clear fluid from the base of the inclined wall reaches its height of neutral buoyancy. In our experiment, the primary intrusion height was unaffected by the presence of weak polydispersity in the system.

Relatively small secondary layers subsequently developed above the primary layer. Consideration of the evolution of the Boycott layer indicates why the secondary layers differ in scale from the primary layer. The primary intrusion comprises clear neutrally buoyant fluid that has risen from the base of the tank. The subsequent evolution of the primary intrusion is driven by the continuing supply of clear fluid from the base of the tank. When the primary intrusion is initiated, clear fluid in the overlying Boycott layer is also neutrally buoyant, as it too has risen through a vertical height $h_{n}$. It is therefore feasible for an intrusion to develop anywhere along the length of this clear fluid layer, and a boundary-layer instability is responsible for setting the initial length scale of the secondary layers. The precise mechanism underlying this instability remains to be determined.

A situation similar to the stratified Boycott effect arises in salt-stratified systems with heated vertical sidewalls (Thorpe et al. 1969; Chen et al. 1971), wherein buoyant up-welling is driven by the sidewalls. Once fluid from the base of the tank reaches its neutral buoyancy height, a primary intrusion develops. At the initiation of the primary intrusion, the overlying boundary-layer fluid is also everywhere neutrally buoyant, and temperature and salinity gradients exist perpendicular to the wall. The resulting sideways diffusive instability (Hart 1971) initially proposed by Thorpe et al. (1969) is then responsible for setting the length scale of secondary intrusions. We could likewise consider the possibility of an instability setting the length scale of the secondary layers in the stratified Boycott effect, using the approaches of Linden \& Weber (1977) and Kerr (1991a). These approaches rest on the assumption of unidirectional flow, and have been successful for double-diffusive systems. Such an assumption is not universally applicable to our system, as the settling of particles away from the wall boundary layer must be balanced by an inflow of fluid to the layer (Acrivos \& Herbolzheimer 1979); this causes the layer thickness to grow with distance along the wall $\left(\delta \sim x^{1 / 3}\right)$. The resulting flow must therefore depend on the along-the-wall coordinate and cannot adequately be modelled as unidirectional, unless one is suitably distant from the base of the tank. To date, we have been unable to obtain an analytical solution, steady or unsteady, for the base flow in the stratified Boycott effect; consequently, a detailed, stability analysis has not been possible.

Another notable contrast between layering in the stratified Boycott effect and double-diffusive systems concerns the long-term layer dynamics. In the case of a heated sidewall (Thorpe 1969), it is found that successive merging of layers that originate from the double-diffusive instability generates layers that scale as $\Delta \rho_{T} /|\mathrm{d} \rho / \mathrm{d} z|$. In this case, the layer mergings are driven by energy supplied from the heated wall. Given that we observed layer mergings in the stratified Boycott effect, it is conceivable that in the presence of a constant source of energy, such as a steady supply of particles, the secondary layers would ultimately merge to an equivalent steady state. Without a constant supply of particles, however, the experiment runs out of energy before 
a uniform secondary-layer length scale can be achieved. In that regard, our system bears a stronger resemblance to that investigated by Linden \& Weber (1977), in which an inclined sidewall was introduced into a double-diffusive salt-sugar system.

We can obtain a qualitative estimate of the effect of layering on the overall settling time by considering an arrangement of $n$ steady layers, in which the $i$ th layer has a thickness $H_{i}$ and base length $X_{i}$. We assume that the top layer evolves as in the absence of ambient stratification, this assumption being supported by our observations of the Boycott effect in the weak stratification limit. The underlying layers are well-mixed, as suggested by experimental density profiles. The overall settling time of the system is thus obtained by combining the Boycott settling time of each layer. For example, using (3.1) to determine the settling time in each layer for our trapezoidal geometry, and summing the settling time of each layer, yields a total settling time

$$
T=\sum_{i} t_{i}=\sum_{i} \frac{H_{i}}{v_{0}}\left(1-\frac{H_{i}}{2 X_{i}}\right),
$$

where $t_{i}$ is the settling time of the $i$ th layer. The derivation of (4.1) is given in Appendix A. Two limiting cases of interest are $n=1$ and $n \rightarrow \infty$. For $n=1$, there is only one layer and the time taken for all the particles to settle out is simply that due to the homogeneous Boycott effect. For large $n$, the settling time for the system must tend to that of an individual particle in the absence of the Boycott effect: in this limit, the layers become sufficiently small that the Boycott effect cannot appreciably enhance settling within any given layer. Thus, the presence of layering evidently reduces the efficiency of settling, by an amount that depends explicitly on the bounding geometry. As an approximation to our experiment, in which the layers are to some degree unsteady, we deduce that the presence of six layers increases the total settling time by approximately $50 \%$, in good qualitative agreement with experimental observations.

The gravitational potential energy of the suspension served to mix the stratified fluid. The efficiency with which the system converted the gravitational potential energy of the sediment to that of the fluid can be quantified in the following manner. The additional energy due to the presence of particles at the start of the experiment is

$$
\Phi_{p}=\int_{V} \Delta \rho_{p} g z \mathrm{~d} V,
$$

where $g$ is the acceleration due to gravity and $V$ is the total fluid volume. After mixing, the increase in gravitational potential energy of the fluid is

$$
\Phi_{f}=\int_{V}\left(\rho_{f}-\rho_{i}\right) g z \mathrm{~d} V,
$$

where $\rho_{f}(z)$ and $\rho_{i}(z)$ are the initial and final stratifications. The efficiency of the system

$$
E=\frac{\Phi_{f}}{\Phi_{p}}
$$

was approximately $10 \%$ in all experiments considered. We note that much of the energy, $\Phi_{p}$, was dissipated through the action of viscosity.

Our study may alternatively be considered as an investigation of the effect of suspended particulate matter on mixing in a stratified ambient with an inclined sidewall. Phillips (1970) and Wunsch (1970) independently examined the convective motions driven in a stratified ambient by the no-flux condition at an inclined sidewall, and 
considered their role in geophysical phenomena. The resulting boundary-layer flow is opposite in direction and weak relative to those induced by the Boycott effect. The ratio of buoyancy forces due to double-diffusion and the Boycott effect is

$$
\beta=\frac{\rho N^{2} L}{g \Delta \rho_{p}}=\frac{L}{h} \frac{\Delta \rho_{s}}{\Delta \rho_{p}},
$$

where $L=\left(4 v \kappa / N^{2}\right)^{1 / 4}$ is the scale of the boundary layer in diffusion-driven flow (Phillips 1970), $v$ the kinematic viscosity, $\kappa$ the diffusivity of salt and $\Delta \rho_{s}$ the change in density due to salt over the depth of the container, $h$. The Boycott effect will thus dominate the double-diffusive mechanism of Phillips (1970) and Wunsch (1970) in any geophysical or laboratory setting for which $\beta \ll 1$.

The stratified Boycott effect may arise in a variety of geophysical settings. We note that this process works equally well for negatively and positively buoyant particles, and so may be applied, for example, in describing flows near a sloping lower boundary forced by degassing of volatiles in magma chambers. The application of this study to the dynamics of magma chambers is the subject of a recent study (Blanchette, Peacock \& Bush 2003).

\section{Appendix A}

The derivation of (4.1) requires us to consider the $i$ th layer with base length $X_{i}$, height $H_{i}$ and a trapezoidal shape with a $45^{\circ}$ angle on one side and a vertical side at the other. We then track the height of the top interface $H$ using (3.1),

$$
\frac{\mathrm{d} H}{\mathrm{~d} t}\left(X_{i}-H\right)=-v_{0} X_{i} .
$$

From the initial condition that $H(0)=H_{i}$, the height of the suspension satisfies

$$
X_{i} H-\frac{1}{2} H^{2}=-v_{0} X_{i} t+X_{i} H_{i}-\frac{1}{2} H_{i}^{2} .
$$

The time taken for the top interface to reach the bottom is then found, by setting $H=0$, to be

$$
t_{i}=\frac{H_{i}}{v_{0}}\left(1-\frac{H_{i}}{2 X_{i}}\right)
$$

\section{REFERENCES}

Acrivos, A. \& Herbolzheimer, E. 1979 Enhanced sedimentation in settling tanks with inclined walls. J. Fluid Mech. 92, 435-457.

Blanchette, F., Peacock, T. \& Bush, J. W. M. 2003 The Boycott effect in magma chambers. Geophys. Res. Lett. 31, L05611.

Boycotт, A. E. 1920 Sedimentation of blood corpuscles. Nature 104, 532.

Brandt, A. \& Fernando, J. S. 1994 Double-Diffusive Convection. Geophysical Monograph, A G U. Brewer, W. H. 1884 On the subsidence of particles in liquid. Mem. Natl Acad. Sci. USA 3, 165.

Chen, C. F. 1975 Double-diffusive effects in an inclined slot. J. Fluid Mech. 72, 721-729.

Chen, C. F., Briggs, D. G. \& Wirtz, R. A. 1971 Stability of thermal convection in a salinity gradient due to lateral heating. Intl J. Heat Mass Transfer 14, 57-65.

GreEN, T. 1987 The importance of double diffusion to the settling of suspended material. Sedimentology 34, 319-331.

Hart, J. E. 1971 On sideways diffusive instability. J. Fluid Mech. 49, 279-288.

Hart, J. E. 1973 Finite amplitude sideways diffusive convection. J. Fluid Mech. 59, 47-64. 
Hill, W. D., Rothfus, R. R. \& Li, K. 1977 Boundary enhanced sedimentation due to settling convection. Intl J. Multiphase Flow 3, 561-583.

Hosol, A. E. \& Dupont, T. F. 1996 Layer formation in monodispersive suspensions and colloids. J. Fluid Mech. 328, 297-311.

Hoyal, D. C. J. D., Bursik, M. I. \& Atkinson, J. F. 1999 Settling-driven convection: a mechanism of sedimentation from stratified fluids. J. Geophys. Res. 104, 7953-7966.

Huppert, H. E., Kerr, R. C. \& Lister, J. R. 1991 Convection and particle entrainment driven by differential sedimentation. J. Fluid Mech. 226, 349-369.

Huppert, H. E., Kerr, R. C. \& Hallworth, M. A. 1984 Heating or cooling a stable compositional gradient from the side. Intl J. Heat Mass Transfer 27, 1395-1401.

Huppert, H. E. \& Linden, P. F. 1979 On heating a stable salinity gradient from below. J. Fluid Mech. 95, 431-464.

Huppert, H. E. \& Turner, J. S. 1978 On melting icebergs. Nature 271, 46-48.

Huppert, H. E. \& TURner, J. S. 1980 Ice blocks melting into a salinity gradient. J. Fluid Mech. 100, 367-384.

KerR, O. S. 1989 Heating a salinity gradient from a vertical sidewall: linear theory. J. Fluid Mech. 207, 323-352.

Kerr, O. S. 1991a Double-diffusive instabilities at a sloping boundary. J. Fluid Mech. 225, 333-354.

KerR, R. C. $1991 b$ Erosion of a stable density gradient by sediment-driven convection. Nature 353, 423-425.

KerR, O. S. 2000 The criteria for the onset of double-diffusive instabilities at a vertical boundary. Phys. Fluids 12, 3289-3292.

Leung, W. F. \& Probstein, R. F. 1983 Lamella tube settlers. Model and Operation. Ind. Engng Chem. Proc. Des. Dev. 22, 58-67.

Linden, P. F. \& Weber, J. E. 1977 The formation of layers in a double-diffusive system with a sloping boundary. J. Fluid Mech. 81, 757-773.

Mendenhall, C. E. \& Mason, M. 1923 The stratified subsidence of fine particles. Proc. Natl Acad. Sci. 9, 199-202.

Mueth, D. M., Crocker, J. C., Esipov, S. E. \& Grier, D. G. 1996 Origin of stratification in creaming emulsions. Phys. Rev. Lett. 77, 578-581.

NaKamura, H. \& Kuroda, K. 1937 La cause de l'acceleration de la vitesse de sedimentation des suspensions dans les recipients inclines. Keijo J. Med. 8, 256-296.

Narusawa, U. \& SuZuKawa, Y. 1981 Experimental study of double-diffusive cellular convection due to a uniform lateral heat flux. J. Fluid Mech. 113, 387-405.

Oster, G. 1965 Density gradients. Sci. Am. 212, 70-76.

Paliwal, R. C. \& Chen, C. F. 1980a Double-diffusive instability in an inclined fluid layer. Part 1. Experimental investigation. J. Fluid Mech. 98, 755-768.

Paliwal, R. C. \& Chen, C. F. $1980 b$ Double-diffusive instability in an inclined fluid layer. Part 2. Stability analysis. J. Fluid Mech. 98, 769-786.

Parsons, J. D., Bush, J. W. M. \& Syvitski, J. P. 2001 Hyperpycnal plume formation from riverine outflows with small sediment concentrations. Sedimentology 48, 465-478.

Phillips, O. M. 1970 On flows induced by diffusion in a stably stratified fluid. Deep-Sea Res. 17, $435-443$.

Ponder, E. 1925 On sedimentation and rouleaux formation. Q. J. Exp. Physiol. 15, 235-252.

Richardson, J. F. \& ZAKI, W. N. 1954 Sedimentation and fluidisation I. Trans. Inst. Chem. Engng 32, 35 .

Schmitt, R. W. 1994 Double diffusion in oceanography. Annu. Rev. Fluid Mech. 26, 255-285.

SiANO, D. B. 1979 Layered sedimentation in suspensions of monodisperse spherical colloidal particles. J. Colloid Interface Sci. 68, 111.

Suzukawa, Y. \& Narusawa, U. 1982 Structure of growing double-diffusive convection cells. Trans. ASME C: J. Heat Transfer 104, 248-254.

TANNY, J. \& TsinOBER, A. B. 1988 The dynamics and structure of double-diffusive layers in sidewall heating experiments. J. Fluid Mech. 196, 135-156.

Thangam, S., Zebib, A. \& Chen, C. F. 1981 Transition from shear to sideways diffusive instability in a vertical slot. J. Fluid Mech. 112, 151-160. 
Thorpe, S. A., Hutt, P. K. \& Soulsby, R.1969 The effect of horizontal gradients on thermohaline convection. J. Fluid Mech. 38, 375-400.

TuRnER, J. S. 1968 The behaviour of a stable salinity gradient heated from below. J. Fluid Mech. 33, 183-200.

Turner, J. S. 1974 Double-diffusive phenomena. Annu. Rev. Fluid Mech. 6, 37-56.

TuRner, J. S. 1980 A fluid-dynamical model of differentiation and layering in magma chambers. Nature 285, 213-215.

Turner, J. S. 1985 Multicomponent convection. Annu. Rev. Fluid Mech. 17, 11-44.

Turner, J. S. \& Chen, C. F. 1974 Two-dimensional effects in double diffusive convection. J. Fluid Mech. 63, 577-592.

Wuest, A. \& CARMaCK, E. 2000 A priori estimates of mixing and circulation in the hard-to-reach water body of lake Vostok. Ocean Modeling 2, 29-43.

Wunsch, C. 1970 On oceanic boundary mixing. Deep-Sea Res. 17, 293-301. 